\title{
Impact of mobile subscribers dual stack IPv4/IPv6 deployment
}

\author{
Mohammad Dian Pahlevia ${ }^{\mathrm{a} \mathrm{b}^{*}}$, Benfano Soewito ${ }^{\mathrm{a}}$ \\ ${ }^{a}$ Computer Science Department, BINUS Graduate Program - Master of Computer Science, Bina Nusantara University, Jakarta, Indonesia 11480 \\ ${ }^{b}$ Core Network Planning Department, Mobile Telecommunication Company, Riyadh, Saudi Arabia 11351
}

Article history:

Received: 4 May 2018 / Received in revised form: 26 May 2018 / Accepted: 29 May 2018

\begin{abstract}
The use of CGNAT at PT. ZYX as a mobile telecommunication service provider cannot be relied as the solution to solve addressing needs against subscriber growth in future technology. Meanwhile, native IPv6 deployment is currently application-driven, which requires maturity support in either subscriber user equipment, network, and application itself. IPv4/IPv6 dual stack deployment was selected by PT. ZYX as stepping stone towards native IPv6 deployment. This paper analyzes the impact of dual stack IPv4/IPv6 deployment for mobile subscribers at PT. ZYX. After selecting the dual stack approach and completing the deployment, test and measurements were performed to confirm the connectivity also against the performance and node utilization to conclude the impact. The test confirmed successful connectivity and the measurements showed that the deployment gives significant enhancement of routing table size and NAT table in node utilization and does not cause performance drop of hop count, throughput, and download time.
\end{abstract}

Keywords: Mobile subscribers; IPv4/IPv6 dual stack; deployment; connectivity; performance; node utilization

\section{Introduction}

At 14 September 2012, Réseaux IP Européens Network Coordination Centre (RIPE NCC) as Regional Internet Registry (RIR) for Europe, Middle East and Central Asia began to allocate IPv4 address space from the last /8 of IPv4 address space it holds [1].

In order to cater subscriber growth with public Internet Protocol version 4 (IPv4) limitation, addressing solution for their subscribers was mandatory for PT. ZYX to guarantee their business continuity. PT. ZYX must choose whether to go with single IPv6, dual stack, or to stay with current Carrier Grade Network Address Translation (CGNAT) solution.

Single Internet Protocol version 6 (IPv6) means assigning single IPv6 address to subscribers' user equipment. This approach requires translation [2] for IPv6 to IPv4 communication or tunneling [2] for IPv6 traversing IPv4 domain in its intermediate path. Dual stack means providing complete support for both IPv4 and IPv6 in hosts and routers [3] in Network Layer to allow both stack to communicate to their peer IP version counterpart independently. Staying with CGNAT solution means translating IPv4 to other IPv4 to save the use of existing limited public IPv4.

Subscriber address is the address assigned by service provider access point either mobile Packet Gateway (PGW), Broadband Network Gateway (BNG), or evolved Packet Data Gateway (ePDG). This address is used as user plane in access network before the access point but is used as both control

* Corresponding author.

Email:pahlev@gmail.com plane and user plane in IP network after the access point.

Fig. 1 shows the architecture of single stack and dual stack IP protocol in Open System Interconnection (OSI) Network Layer.

PT. ZYX currently has license to sell both fixed and mobile network services through Fiber to the Home (FTTH) FTTH, Dedicated Internet Access (DIA), Wireless Fidelity (WiFi) offload, and $2 \mathrm{G} / 3 \mathrm{G} / 4 \mathrm{G}$ cellular networks but the deployment will be applied only to mobile network services. Fig. 2 shows $4 \mathrm{G}$ user plane architecture.

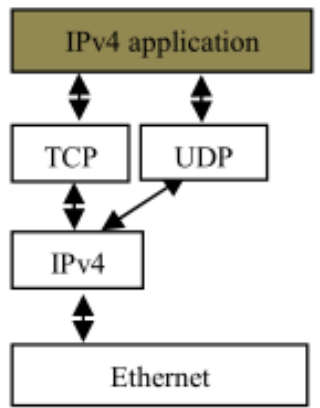

a) Single Stack

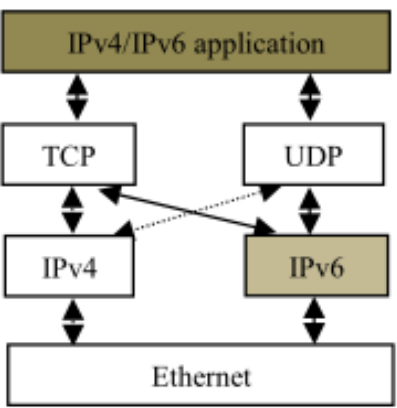

b) Dual Stack
Fig. 1. Single and dual stack IP version architecture at OSI network layer [4]

From mobile 3G Partnership Project (3GPP) control plane point of view, PDPType or PDNType parameter is negotiated either to use type IPv4, IPv4v6 or IPv6 as shown in Fig. 3. Only Stateless Address Auto Configuration (SLAAC) is supported by 3G Partnership Project (3GPP) [6]. A Mobile 
Packet Gateway (PGW) assigns a /64 IPv6 address dynamically to the User Equipment (UE) as its Packet Data Protocol (PDP) address s specified in Internet Engineering Task Force (IETF) Request for Comment (RFC) 3314 [7]. The type IPv4v6 defined after 3GPP Release 9 which optimize the utilization of Packet Data Protocol (PDP) context for dual stack scenario. Table 1 shows the difference between dual stack implementation before and after 3GPP Release 9 as mentioned by Korhonen [6].

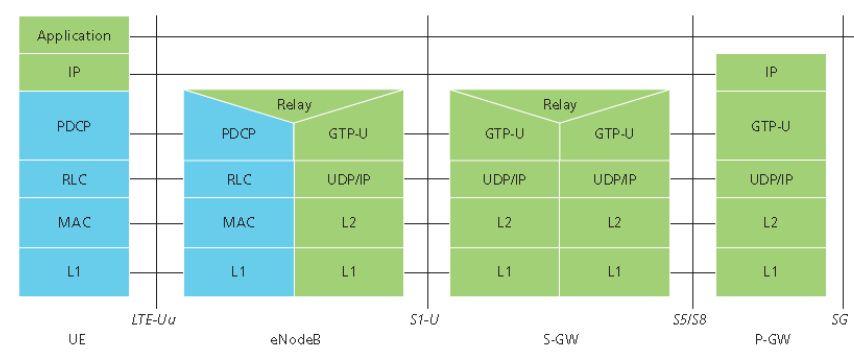

Fig. 2. 4G User plane architecture [5]

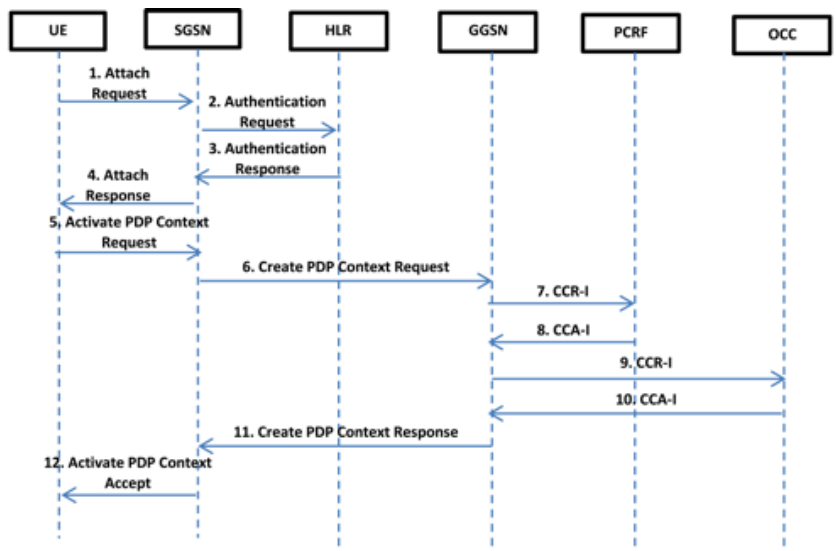

(a)

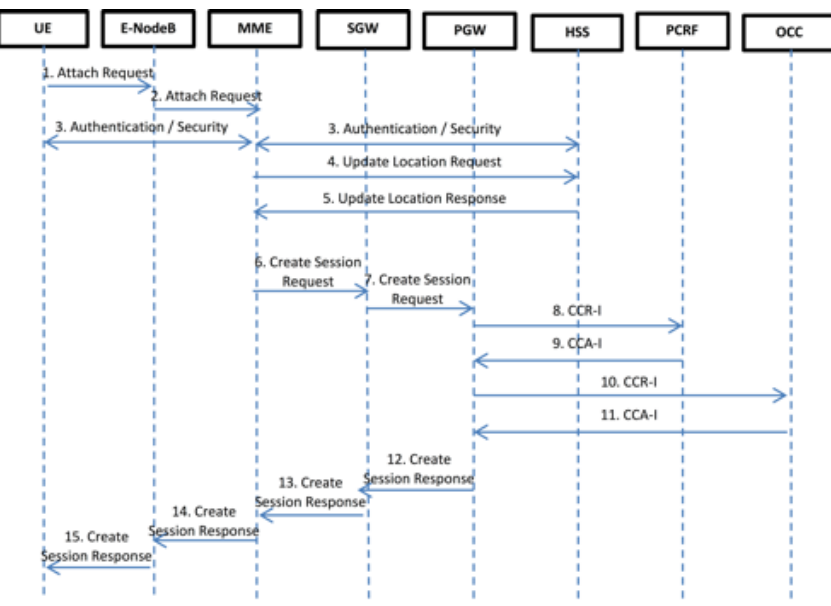

(b)

Fig. 3. Data Call Setup Flow: (a) 2G/3G; (b) 4G

For single stack communication, single PDP address is used for all subscriber communication. For dual stack communication, address selection algorithm need to be considered to ensure optimum use of IPv6 stack and IPv4 offload at the meantime. There are two available algorithms to ensure the preference use of IPv6 over IPv4.
Table 1. Dual stack PDP context comparisons before and after 3GPP R9

\begin{tabular}{|c|c|c|c|}
\hline $\begin{array}{c}\text { 3GPP } \\
\text { version }\end{array}$ & $\begin{array}{l}\text { No. of } \\
\text { context }\end{array}$ & PDPType & PDP Address \\
\hline $\begin{array}{l}\text { Before } \\
\text { R9 }\end{array}$ & 2 & $\begin{array}{l}\text { One context with } \\
\text { PDPType = IPv4 } \\
\text { and } \\
\text { One context with } \\
\text { PDPType = IPv6 }\end{array}$ & $\begin{array}{l}\text { Single address per } \\
\text { context. }\end{array}$ \\
\hline $\begin{array}{l}\text { R9 and } \\
\text { after }\end{array}$ & 1 & $\begin{array}{l}\text { One context with } \\
\text { PDPType = IPv4v6 }\end{array}$ & $\begin{array}{c}\text { Two addresses per } \\
\text { context }\end{array}$ \\
\hline
\end{tabular}

First algorithm was defined by Thaler [8] which is currently appointed as an IETF standard RFC 6724 and implemented by default in a host. This algorithm specifies address selection behavior for all IPv6 implementation. If there is Source A (SA) and Source B (SB) in a host interface address trying to communicate with Destination (D), also if there are Destination A (DA) and Destination B (DB) exist with Source(A) and Source(B) are their respective source address. The default policy table of this algorithm gives IPv6 addresses higher preferences than IPv4 address.

For example, if we have candidate source addresses: 2001:db8:1::2 or fe80::1 or 169.254.13.78, candidate destination address list 2001:db8:1::1 or 198.51.100.121, then the default behavior result will select 2001:db8:1::1 as the destination with 2001:db8:1::2 as source address then 198.51.100.121 with 169.254.13.78 as source address (prefer matching scope) if first alternative fails. This algorithm aware about unwanted timeout at broken IPv6 condition and suggest to alter the default policy by preferring IPv4 in the hardware or software implementation [8].

Second algorithm is called Happy Eyeballs algorithm defined by Wing and Yourtchenko [9]. It is appointed as RFC 6555 which allows a dual stack host to maintain its quality compared to IPv4-only host by improving Transmission Control Protocol (TCP) connect time. TCP connect time improvement can be achieved by reducing TCP connect timeout in the orders of tens of milliseconds. Fig. 4 shows the Happy Eyeball algorithm scenario. This algorithm has been tested by Bajpai and Schonwalder [10] and showing improvement in TCP Connect Time by using Mozilla Firefox v15, Google Chrome 11, Opera 12.10 and Apple Safari on OS X 10.11 and Apple IOS 9.

Bajpai and Schronwalder use source address selection order then let IPv6 connection to start $300 \mathrm{~ms}$ ahead to give it fair chance to succeed. Bajpai and Schonwalder conclude that TCP connect times to popular dual stacked websites over IPv6 have improved over time. As of May 2016, 18\% of the top 10K ALEXA websites are faster over IPv6 while $91 \%$ of the rest are at most 1 millisecond slower. A 300 milliseconds timer value therefore leaves only around $2 \%$ chance for IPv4 to win a HE-race to these websites. In $90 \%$ of these cases, HE tends to prefer slower IPv6 connection, although the TCP connect times are not that far apart from IPv4. We showed that a HE timer value of 150 milliseconds provides a margin benefit of $10 \%$ while retaining similar IPv6 preference levels for $99 \%$ of the dual-stacked websites [10].

As per above references, User Equipment (UE) is expected to use IPv6 for their communication and reduce the IPv4 session table usage. The references mentioned above also concluded that dual stack IPv4/IPv6 is complied with the 
requirement to be implemented in PT. ZYX network.

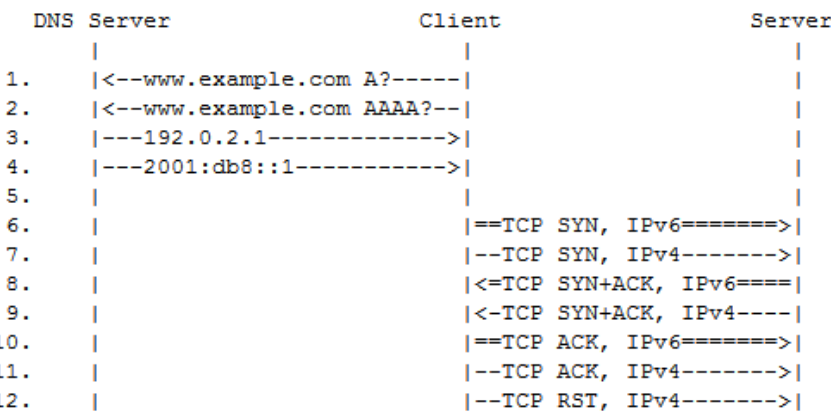

(a)

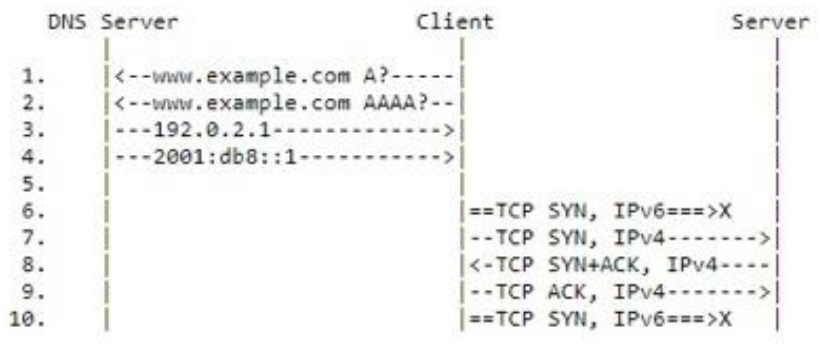

(b)

Fig. 4. Happy Eyeball Scenario: (a) Successful IPv6; (b) Broken IPv6 [9]

Another work as presented in [11] said that the IPv6 network is able to provide stable network connectivity for IPv6 end-hosts. Due to the relatively light traffic load and abundant bandwidth in the IPv6 backbone, the IPv6 throughput is easily superior to that of IPv4.

By performing this deployment and measurements of some performance indicators, mobile providers will have reference whether implementing dual stack IPv4/IPv6 results in enhancement or performance drop.

\section{Materials and Methods}

\subsection{Deployment}

This case study deployment is based on conceptual framework as described in Fig. 5. The deployment follows Prepare, Planning, Design, Implement, Operate, and Optimize (PPDIOO) [12] framework to assess and distinguish proper step of deployment.

This case study deployment changed only subscribers address part and any related entity which use subscriber address as attribute in their communication. Any remaining unrelated entity in the network will remain unchanged to reduce complexity. Table 2 lists PT. ZYX network domain and whether changes were required there.

Furthermore, Table 3 lists node level changes which were required to deploy IPv4/IPv6 dual stack at PT. ZYX. From Table 2 and Table 3, there is no additional hardware required as well as hardware change. No change on traffic flow as well. Furthermore, migration steps were planned to meet minimum subscriber involvement.

\subsection{Tests and Measurements}

After successful deployment, measurements were taken in live network to obtain real value of the desired Key Performance Indicator (KPI)

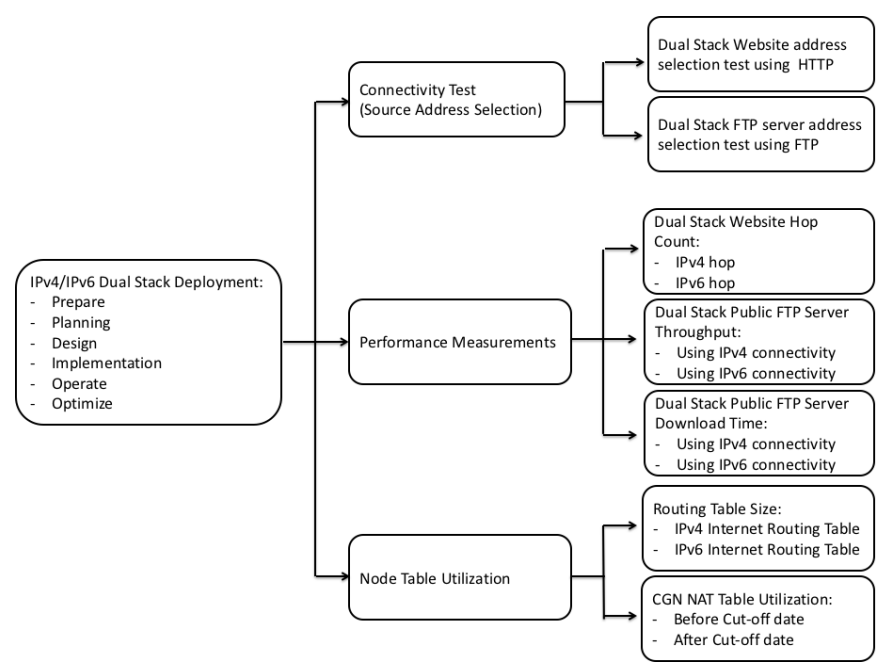

Fig. 5. Deployment and Measurement Flowchart

Table 2. PT ZYX network domain

\begin{tabular}{ccc}
\hline Domain & $\begin{array}{c}\text { Mobile Subscriber Dual } \\
\text { Stack Path } \\
\text { (Yes/No) }\end{array}$ & $\begin{array}{c}\text { Required } \\
\text { Change } \\
\text { (Yes/No) }\end{array}$ \\
\hline $\begin{array}{c}\text { Yes } \\
\text { Mobile Radio Access }\end{array}$ & No \\
Network & No & No \\
Mobile Packet Core & Yes & Yes \\
MPLS & Yes & Yes \\
Charging and Billing & No & Yes \\
Corporate Customers & No & No \\
GPRS Roaming Exchange & No & No \\
Internet Gateway (ISP) & Yes & Yes \\
Information Technology & No & No \\
(IT) Department & No & No \\
Value Added Service & & No \\
(VAS) & No & No \\
Network Operation Center & & No \\
(NOC) & No & No \\
Contact Center & No & No \\
Corporate & &
\end{tabular}

As shown in Fig. 6, $\mathrm{A}$ is a dual stack subscriber UE which having both usable IPv4 and IPv6 as its PDP Address to connect to other entities using IPv4 link and IPv6 link. B is the PT. ZYX dual stack network. B can be broken down into $\mathrm{Ba}$ and $\mathrm{Bb}$ which represent dual stack network elements inside PT. ZYX network. $\mathrm{C}$ is a dual stack upstream destination outside PT. ZYX network which is reachable through IPv4 link and IPv6 link. C can be PT. ZYX's upstream provider or a dual stack destination server in the internet.

First test is connectivity test (Fig. 7) This test has two objectives. First objective is to prove subscriber UE connectivity to PT. ZYX mobile network. Successful connectivity means the subscriber is successfully pass all the signaling part with dual stack parameters inside the signaling packet. Second objective is to prove the use of IPv6 PDP Address towards http://ripe.net and $\mathrm{ftp}: / /$ speedtest.tele2.net as sample dual stack destinations. This will also prove default address selection algorithm in subscribers UE.

Second test is hop count test (Fig. 8). This test compares IPv4 and IPv6 path towards dual stack service in the internet. 
This test is taken in order to prove IPv4 and IPv6 path congruency towards a real end location of dual stack destination. As the deployment goal is to make congruent path inside PT. ZYX network, the test took place in the border routers towards outside dual stack destination.

Table 3. Node level change requirement

\begin{tabular}{|c|c|c|}
\hline Domain & Network Element & Required Change \\
\hline Mobile Packet & Serving GPRS Support & License activation \\
\hline \multirow[t]{2}{*}{ Core } & $\begin{array}{l}\text { Node-Mobility } \\
\text { Management Entity } \\
\text { (SGSN-MME) }\end{array}$ & Software configuration \\
\hline & $\begin{array}{c}\text { Serving Gateway /Packet } \\
\text { Gateway (SGW/PGW) }\end{array}$ & $\begin{array}{l}\text { Dual stack software } \\
\text { configuration }\end{array}$ \\
\hline $\begin{array}{l}\text { Multiprotocol } \\
\text { Label } \\
\text { Switching } \\
\text { (MPLS) }\end{array}$ & $\begin{array}{l}\text { Provider Edge }(\mathrm{PE}) \\
\text { Routers }\end{array}$ & $\begin{array}{l}\text { Dual stack software } \\
\text { configuration }\end{array}$ \\
\hline Charging and & Charging System & Software upgrade \\
\hline Billing & Mediation & $\begin{array}{l}\text { Software downgrade } \\
\text { Configuration adaptation }\end{array}$ \\
\hline \multirow[t]{7}{*}{$\begin{array}{c}\text { Internet } \\
\text { Gateway (ISP) }\end{array}$} & Border Routers & $\begin{array}{l}\text { Dual stack software } \\
\text { configuration }\end{array}$ \\
\hline & Service Switches & $\begin{array}{l}\text { Dual stack software } \\
\text { configuration }\end{array}$ \\
\hline & Domain Name Servers & $\begin{array}{l}\text { Dual stack software } \\
\text { configuration }\end{array}$ \\
\hline & Web Filtering & $\begin{array}{l}\text { Dual stack software } \\
\text { configuration }\end{array}$ \\
\hline & Content Delivery Nodes & $\begin{array}{l}\text { Dual stack software } \\
\text { configuration }\end{array}$ \\
\hline & $\begin{array}{l}\text { Anti-Distributed Denial } \\
\text { of Service (DDoS) }\end{array}$ & $\begin{array}{l}\text { Dual stack software } \\
\text { configuration }\end{array}$ \\
\hline & CGNAT & $\begin{array}{l}\text { Dual stack software } \\
\text { configuration }\end{array}$ \\
\hline
\end{tabular}

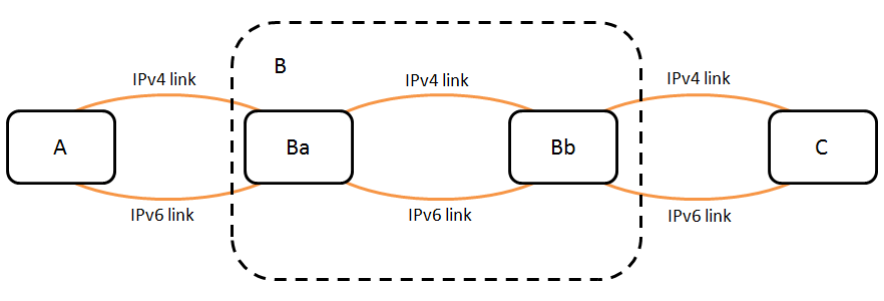

Fig. 6 Measurement model

The dual stack destinations are taken from web daily Alexa Top website, obtained from [13] list. If traceroute is failed due to website owner's policy restriction, additional website is taken from [14]. Successful traceroute must meet the criteria that both IPv4 and IPv6 addresses must be traceable. Secondly, traceroute must reach the destination IP, not end at another IP and the last criteria is the dual stack website must not be located within CDN or public hosting such as Akamai, Google, Cloudflare, Amazon and other similar brands.

Referring to Fig. 6, the real test environment topology is shown in Fig. 8. SecureCRT is installed in MAC Book Pro. MAC Book Pro is used as end user terminal which contacted PT. ZYX Border Router using Secure Shell (SSH). From Border Router, the test was performed towards destination sites.

Third test is throughput test. This test compares amount of transferrable traffic from dual stack services to subscriber UE through FTP service. FTP was used to ensure the service is terminated at real location without content caching at content delivery nodes $(\mathrm{CDN})$.

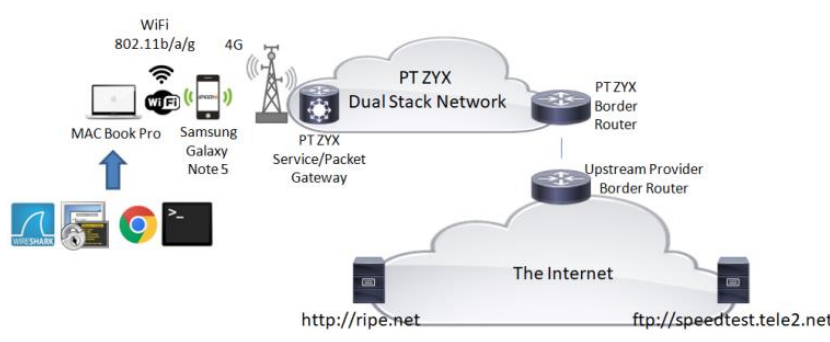

Fig. 7. Connectivity test environment
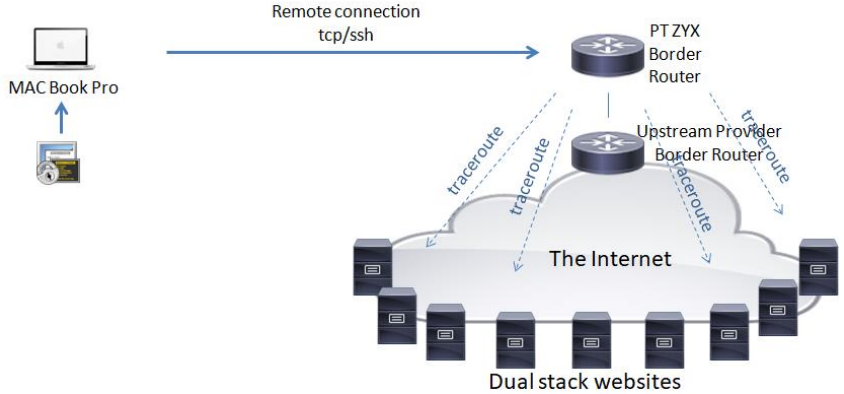

Fig. 8. Hop count test environment

Fourth test is download time test. This test compares download time towards dual stack destinations. To get end-toend value, the measurement took place in the sample dual stack subscriber UE.

Both throughput and download time test are using the same topology and measurement. The tests were performed at dual stack subscriber UE by downloading 10MB file from dual stack FTP server using each IPv4 and IPv6 link towards Tele2 public FTP server IPv4 address 90.130.70.73 and IPv6 address 2a00:800:1010::1. The tests were performed during peak and off-peak hour for three weeks. Each measurement recorded average value of 30 times throughput and download time value for each IPv4 and IPv6.

Referring to Fig. 6, the real test environment topology for throughput and download time is shown in Fig. 9. vsFTPd was used as FTP client in MAC Book Pro. MAC Book Pro connected to Samsung Galaxy 5 over WiFi then Samsung Galaxy 5 as the modem connected to PT. ZYX 4G mobile network to reach the FTP server.

Fifth test is routing table size test. This test compares routing table size between IPv4 and IPv6 routing table. This test is taken in order to prove the IPv6 advantage of addressing hierarchy. This test took place in the border routers as these routers accept internet routing tables from upstream provider.

As per measurement model shown in Fig. 6, the measurement topology was set up as per Fig. 10. SecureCRT which is installed in MacBook Pro was used as SSH client towards border routers. Secure Shell (SSH) operations were performed to get the value from border routers.

Sixth test is CGNNAT table utilization. This test is a measurement which compares the utilization of IPv4 NAT table one month before until nine months after the deployment. This measurement is taken to prove source address selection and Happy Eyeballs algorithms in subscribers UE. CGN is the network element which the measurement takes place. As per measurement model in Fig. 
6, the measurement topology was set up and is shown in Fig. 11. CGNAT plays role as B and sits between subscribers and the Internet.

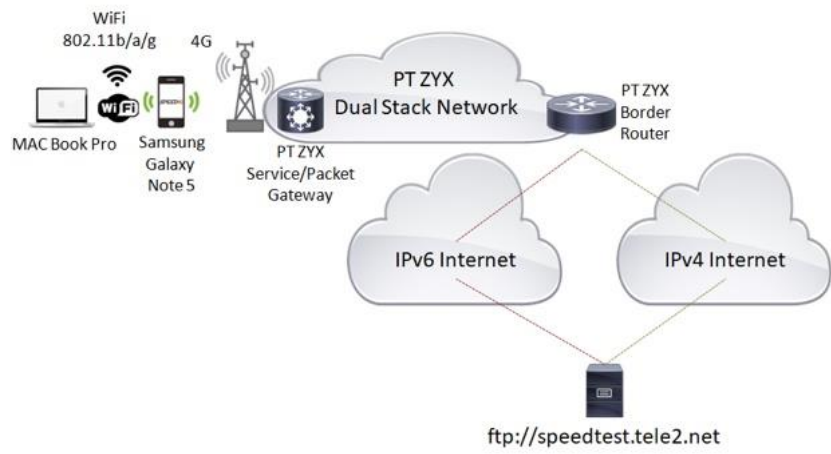

Fig. 9. Throughput and download time test environment

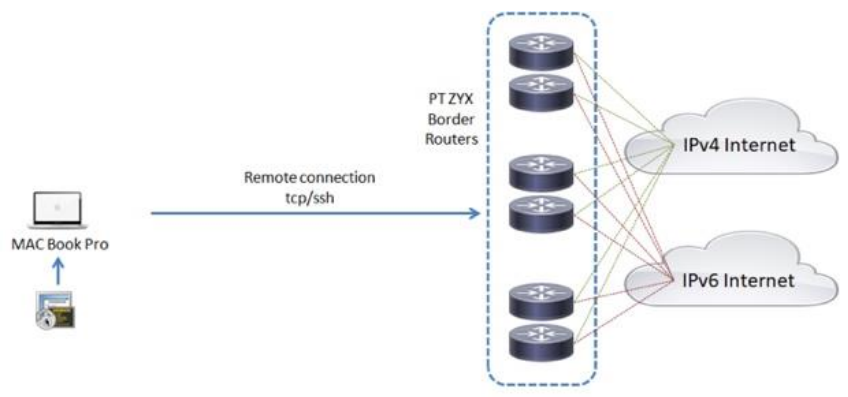

Fig. 10. Routing table size test environment

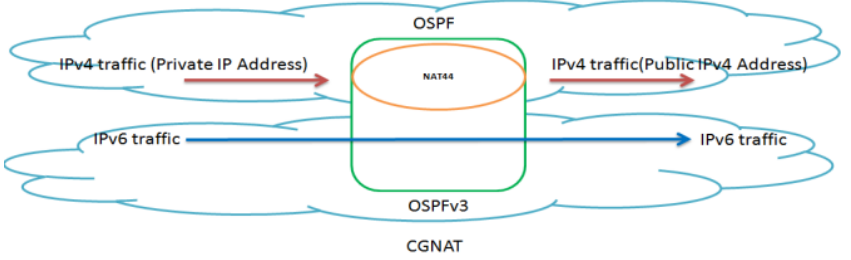

Fig. 11. CGNAT with dual stack traffic architecture

\section{Results and Discussion}

\subsection{Connectivity Test}

IPv6 Hypertext Transfer Protocol (HTTP) connectivity was successfully established between dual stack subscriber UE and sample HTTP website http://ripe.net. IPv6 File Transfer Protocol (FTP) connectivity also successfully done towards sample FTP website ftp://speedtest.tele2.net.

Fig. 12 shows that dual stack subscriber UE send both A and AAAA Domain Name Service (DNS) query towards dual stack DNS server requesting IP Addresses of http://ripe.net then the dual stack DNS send both A and AAAA DNS response towards the subscriber. Fig. 13 shows that the subscriber UE send HTTP request using its IPv6 Address.

The dual stack subscriber UE then successfully opened dual stack website http://ripe.net using its IPv6 address. The main page of the http://ripe.net website shows the requestor IP Address on its browser as can be seen in Fig. 14. This figure proves that source address selection prefers IPv6 address for HTTP service as expected in this deployment.

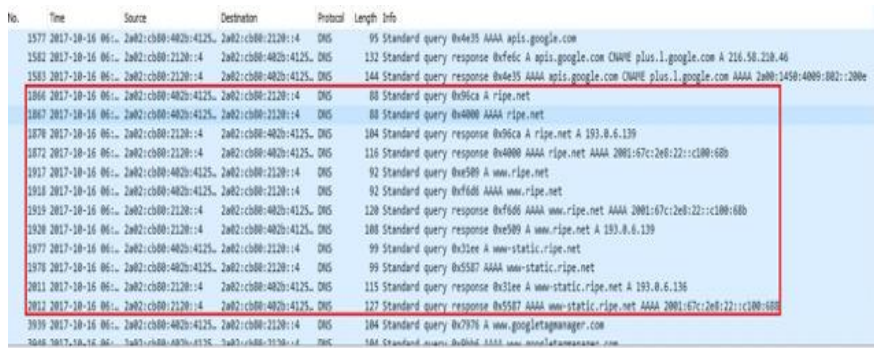

Fig. 12. Dual stack DNS query and response trace

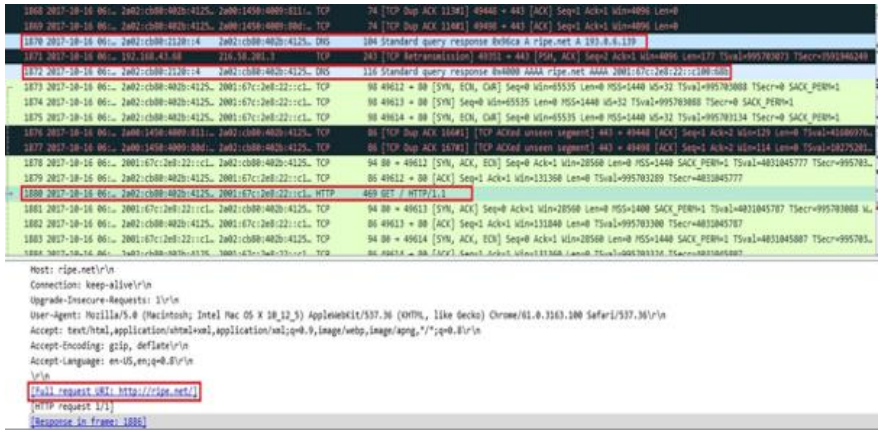

Fig. 13. Dual stack source address selection trace

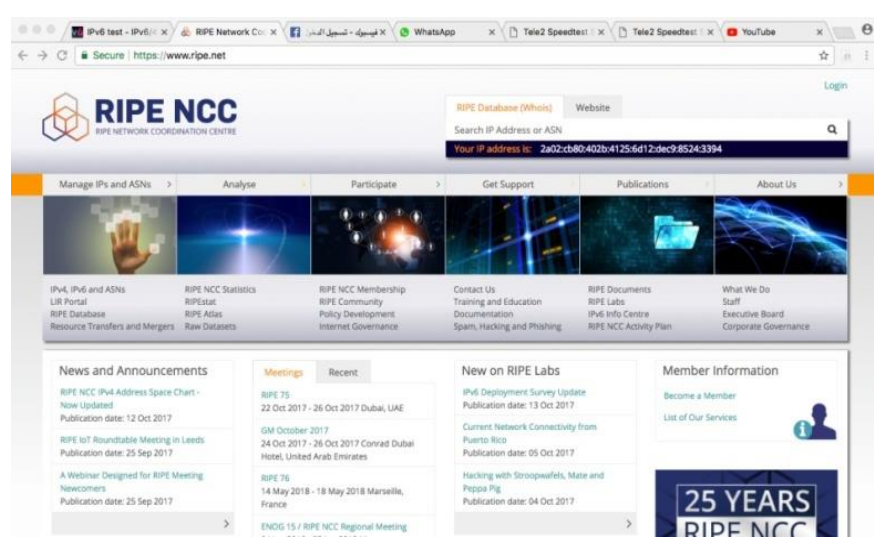

Fig. 14. Source address resolution at destination website

For FTP test, it is shown in Fig. 15 that when dual stack subscriber UE send FTP towards ftp://speedtest.tele2.net using hostname, the dual stack FTP server responds with its IPv6 address. This figure proves that source address selection prefers IPv6 address for FTP service as expected in the deployment.

\subsection{Performance Measurement}

From the dual stack website list which meet criteria as mentioned in Section 2.2, 45\% websites from RIPE NCC shows closer IPv6 hop and 29\% shows equal IPv6 hop away from PT. ZYX border routers. From American Registry for Internet Numbers (ARIN) region, 39\% of them have better IPv6 hop and $18 \%$ of them have equal hop count. Asia Pacific Network Information Centre (APNIC) region has 37\% closer location in IPv6 and 13\% equal hop. Latin America and Caribbean Network Information Centre (LACNIC) region has only three dual stack websites which meet criteria and all of them has closer IPv6 location compared to its IPv4 location away from PT. ZYX border routers. Fig. 16 shows traceroute 
result of dual stack websites which meets the criteria from all regions.

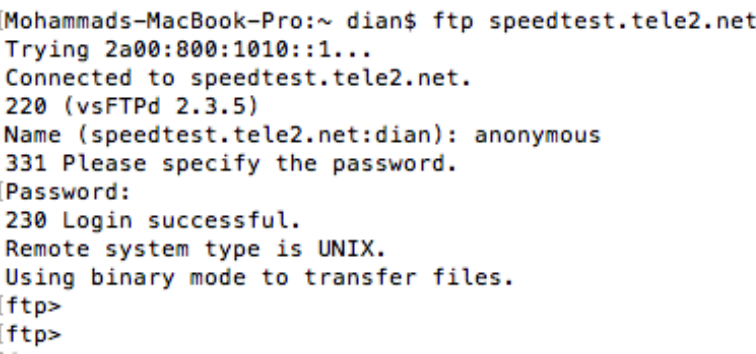

Fig. 15. FTP source address selection result

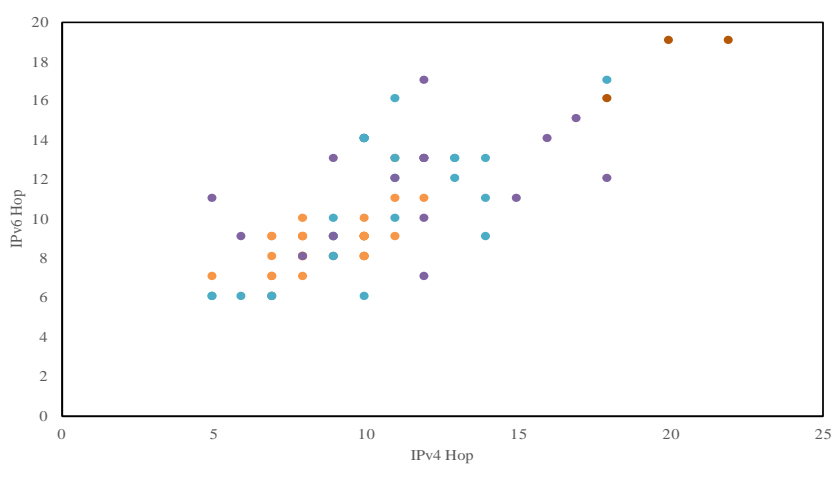

$\bullet$ RIPE NCC • ARIN • APNIC • LACNIC

Fig. 16. Hop count test result

Figs. 17 and 18 show average value of the three-week test result during off-peak and peak hour. The test was performed in the live network at real network condition in order to get real subscriber experience. There was some intermittent condition where the modem fell back to $3 \mathrm{G}$ then came back to 4G. There was also condition where file transfer was stable but with small throughput. The test was not repeated unless for stalled or modem disconnection state.

The average throughput value of daily test result was taken and it gives us 2\% IPv6 better throughput performance during off-peak hour meanwhile IPv4 gives better $4 \%$ throughput during peak hours. Fig. 17 shows throughput test result.

\subsection{Node Utilization Table}

From routing table perspective, the amount of IPv6 routes received from External order Gateway Protocol (EGBP) at PT. ZYX border routes only $6 \%$ of IPv4 received routes. Fig. 19 shows average of Routing Table Size received from Internet for both IPv4 and IPv6.

On CGNAT NAT table, the daily monitoring of total IPv4 session from one month before cut-off date up to nine months afterwards. Fig. 20 shows PT. ZYX CGNAT NAT Table trend.

Referring to Fig. 20, there is linear trend of NAT session table at PT. ZYX CGNAT. In the interval of 1 November 2016 (20161101) to 14 December 2016 (20161214), total session trend in red is increasing due to subscribers and traffic growth. All IPv4 session towards internet will utilize CGNAT NAT table during this period. Starting from $15^{\text {th }}$ Dec 2016 (20161215) which is the cut-off date until end of the graph
(20170927), we can see the decreasing trend of CGNAT NAT total session in blue due to the increasing of IPv6 traffic. IPv6 session will not utilize CGNAT NAT table as the IPv6 address obtained from PGW can be routed directly towards the Internet.

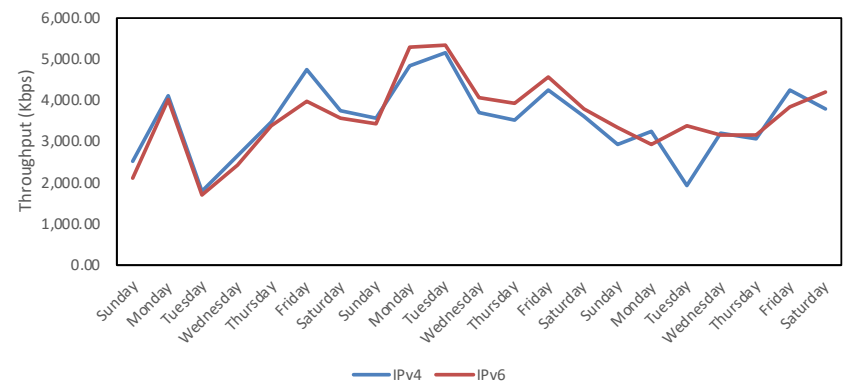

(a)

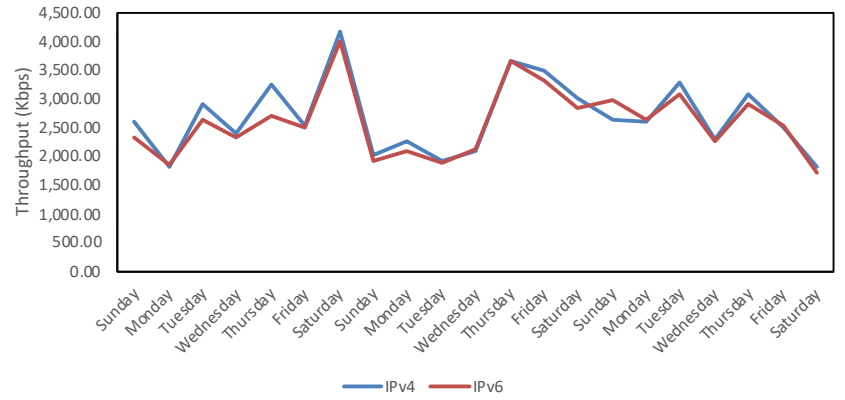

(b)

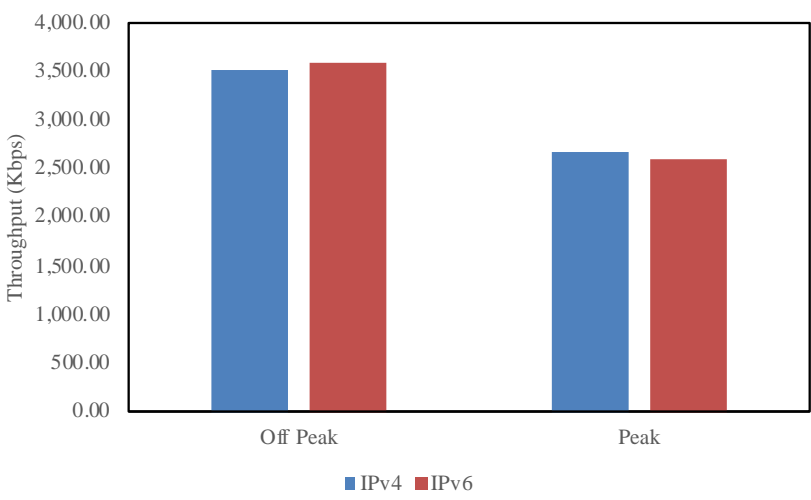

(c)

Fig. 17. Throughput test result: (a) off-peak hour; (b) peak hour; (c) average

The daily monitoring of total IPv4 session from one month before cut-off date up to nine months after shows $69.64 \%$ saving against the trend.

\section{Conclusion}

Dual stack is the best approach deployment for PT. ZYX as it does not require dramatic change in the network such as traffic flow or hardware change. Dual stack also allows existing IPv4 UE to perform connectivity as usual without any impact.

Based on the tests, it is concluded that dual stack deployment at PT. ZYX provide $69.64 \%$ enhancement in CGNAT NAT table compare to its trend. This is due to the source address algorithm which prioritize the use of IPv6 address part by default. IPv6 routing table also shows small 
amounts of routes by having only $6 \%$ routes compare to IPv4 routing table size.

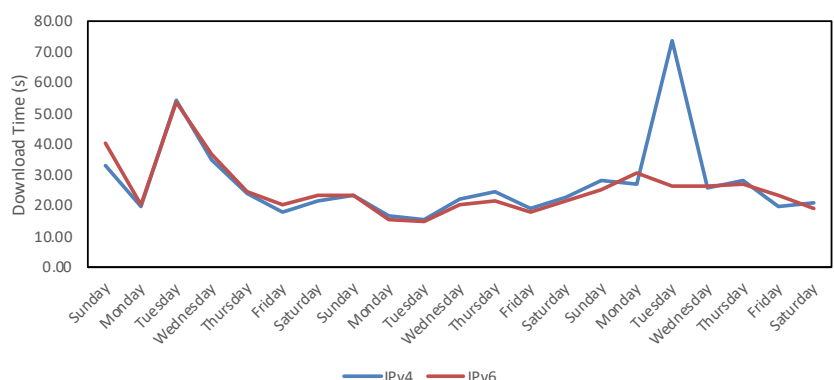

(a)

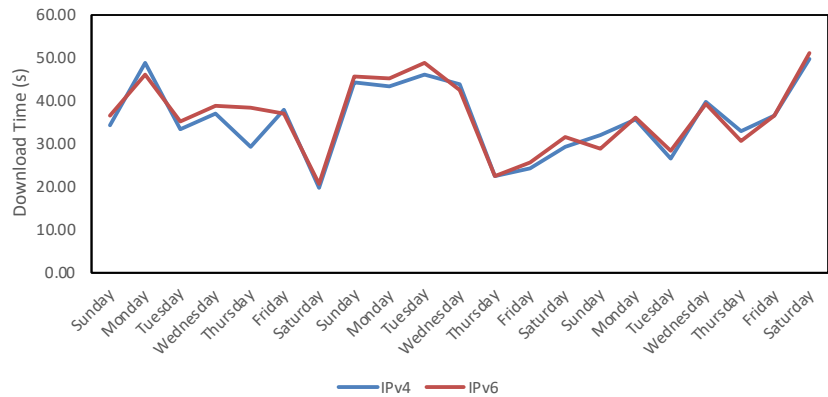

(b)

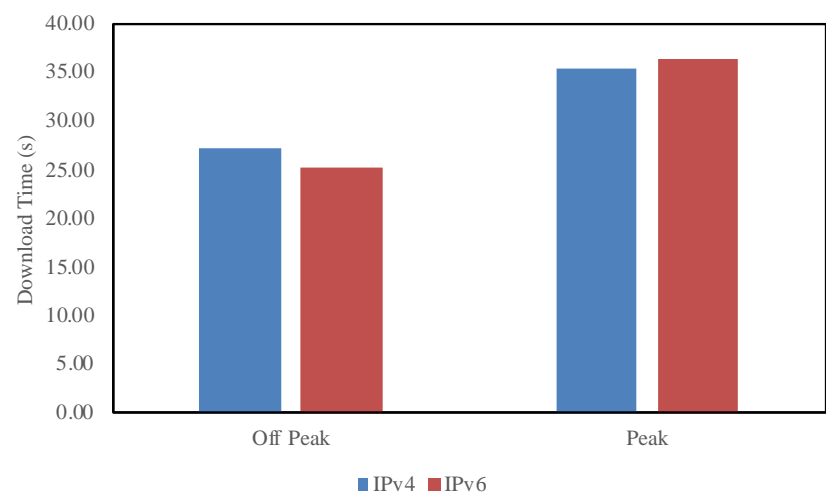

(c)

Fig. 18 Download time test tesult: (a) off-peak hour; (b) peak hour; (c) average

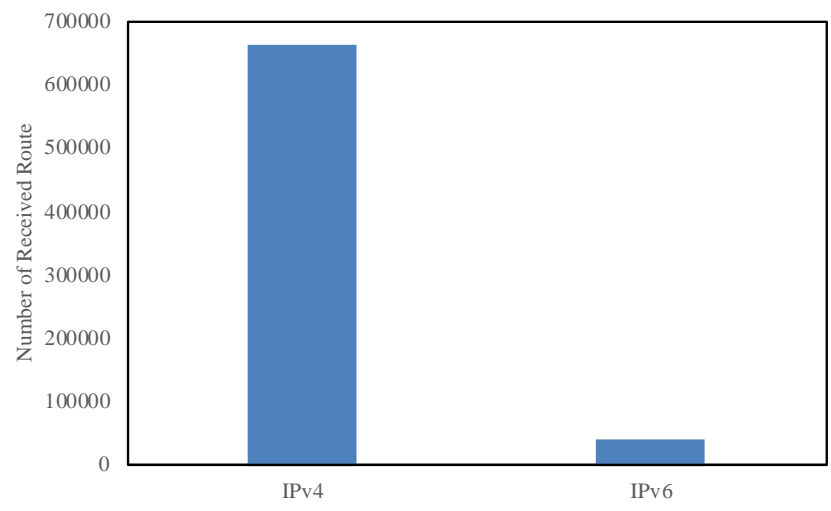

Fig. 19. Routing table size test result

It is seen as well that the deployment does not cause performance drop. Both throughput and download time test shows better IPv6 performance in off peak hour but shows the opposite during peak hour. The hop count test meanwhile shows that having IPv6 does not cause all websites far away to reach from our network compare to their IPv4 location.

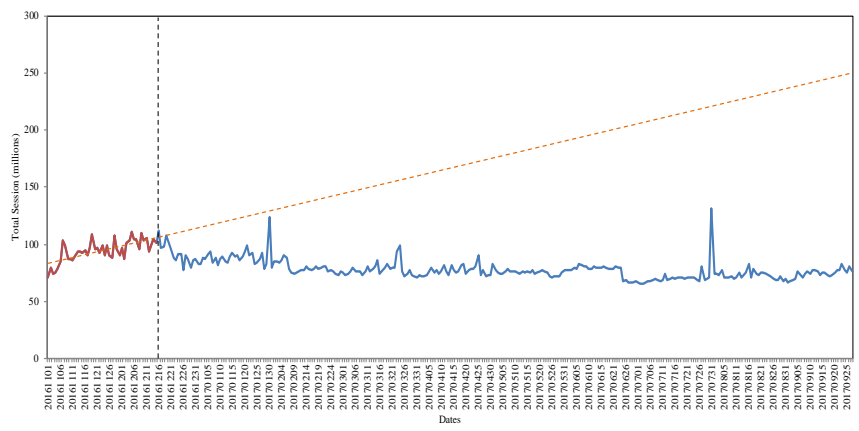

Fig. 20. PT. ZYX CGNAT NAT table trend

Based on above, its concluded that dual stack IPv4/IPv6 deployment study case is mainly solves PT. ZYX IPv4 exhaustion issue by giving significant enhancement in IPv4 CGNAT NAT table utilization also offering small IPv6 routing table size and does not give significant enhancement in more subscriber performance experience.

This case study result influenced by network condition. Network condition could cause anomaly and affect the performance value. Another case study might give different performance value. This case study also did not compare dual stack against tunneling and translation as tunneling and translation implementation require more complex redesign in the network.

For future works, some studies can be performed as next stepping stone to native IPv6 such as adaptive address assignment based on user device type. Another study such as some ad hoc IPv4 address assignment mechanism to assign public IPv4 based on session request can also be considered.

Some comparative study such as the performance comparison against fixed network services with its Broadband Network Gateway (BNG) and its Point to Point Protocol over Ethernet (PPPoE) signaling, or towards some Internet of Things (IoT) use cases also can be appointed as case study.

\section{Acknowledgements}

A special thanks to Core Network Planning Department of PT. ZYX for their support in this study.

\section{References}

1. IPv4 Exhaustion - RIPE Network Coordination Centre, (n.d.). https://www.ripe.net/publications/ipv6-info-centre/about-ipv6/ipv4exhaustion (accessed September 18, 2016).

2. D.G. Waddington, F. Chang, Realizing the transition to IPv6, IEEE Commun. Mag. (2002). doi:10.1109/MCOM.2002.1007420.

3. R.E. Gilligan, E. Nordmark, Basic Transition Mechanisms for IPv6 Hosts and Routers, (2005). doi:10.17487/RFC4213.

4. R.H. Sailan, Mohd. Khairil, Impact of TCP window size on IPv4 and IPv6 performance, Int. J. Comput. Sci. Netw. Secur. 9 (2009) 129--133.

5. Alcatel-Lucent, The LTE Network Architecture, A Comprehensive Tutorial, 2009. 
6. J. Soininen, B. Patil, T. Savolainen, G. Bajko, K. Iisakkila, IPv6 in 3rd Generation Partnership Project (3GPP) Evolved Packet System (EPS), 2012. doi:10.17487/rfc6459.

7. M. Wasserman, ed., Recommendations for IPv6 in Third Generation Partnership Project (3GPP) Standards, 2002. doi:10.17487/rfc3314.

8. R. Draves, A. Matsumoto, T. Chown, Default Address Selection for Internet Protocol Version 6 (IPv6), 2012. doi:10.17487/rfc6724.

9. D. Wing, A. Yourtchenko, Happy Eyeballs: Success with Dual-Stack Hosts, 2012. doi:10.17487/rfc6555.

10. V. Bajpai, Measuring the Effects of Happy Eyeballs Happy Eyeballs Algorithm [ RFC 6555 ], (2013).
11. Y.N. Law, M.C. Lai, W.L. Tan, W.C. Lau, Empirical performance of IPv6 vs. IPv4 under a dual-stack environment, IEEE Int. Conf. Commun. (2008) 5924-5929.

12. B. Sivasubramanian, E. Frahim, R. Froom, PPDIOO Lifecycle Approach to Network Design and Implementation \&gt; Analyzing the Cisco Enterprise Campus Architecture, Anal. Cisco Enterp. Campus Archit. (2010) 37-50. http://www.ciscopress.com/articles/article.asp?p=1608131 \&seqNum=3 (accessed June 13, 2017).

13. IPv6 Status of MOZ.COM Global 500 Websites, (n.d.). http://www.delong.com/ipv6_alexa500.html (accessed June 13, 2017).

14. D. Wing, Weblet Importer, (n.d.). http://www.employees.org / dwing/aaaa-stats/ipv6-aaaa-valid.2017-10-18_0800.txt (accessed October 18, 2017). 